\title{
MIXING FRACTION OF INNER SOLAR SYSTEM MATERIAL IN COMET 81P/WILD2
}

\author{
A. J. Westrhal ${ }^{1}$, S. C. Fakra ${ }^{2}$, Z. Gainsforth ${ }^{1}$, M. A. Marcus ${ }^{2}$, R. C. Ogliore ${ }^{1}$, And A. L. Butterworth ${ }^{1}$ \\ ${ }^{1}$ Space Sciences Laboratory, University of California at Berkeley, Berkeley, CA 94720, USA \\ 2 Advanced Light Source, Lawrence Berkeley National Laboratory, Berkeley, CA 94720, USA \\ Received 2008 April 25; accepted 2008 November 2; published 2009 March 13
}

\begin{abstract}
The presence of crystalline silicates in the comae of comets, inferred through infrared observations, has been a long-standing puzzle. Crystalline silicates are unexpected if comets are composed of pristine interstellar material, since interstellar silicates are almost entirely amorphous. Heating to $>1100 \mathrm{~K}$ can anneal silicates to crystallinity, but no protoplanetary heating sources have been identified that were sufficiently strong to heat materials in the outer nebula to such high temperatures. This conundrum led to the suggestion that large-scale mixing was important in the protoplanetary disk. Reports of refractory calcium-aluminum-rich inclusion-like objects and large concentrations of noble gases in Stardust samples underscore the need for such mixing. However, the evidence from the Stardust samples until now has been largely anecdotal, and it has not been possible to place quantitative constraints on the mixing fraction. Here we report synchrotron-based X-ray microprobe measurements of the relative concentrations of the chemical state of iron in material from a known comet, the Jupiter-family comet $81 \mathrm{P} / \mathrm{Wild} 2$. We find that the comet is rich in iron sulfides. The elemental $\mathrm{S} / \mathrm{Fe}$ ratio based on the sulfide concentration, $\mathrm{S} / \mathrm{Fe}>0.31(2 \sigma)$, is higher than in most chondritic meteorites. We also found that Fe-bearing silicates are at least $50 \%$ crystalline. Based on these measurements, we estimate the fraction $\psi$ of inner nebular material in $81 \mathrm{P} / \mathrm{Wild} 2$. With the lower bound on the crystalline Fe-bearing silicate fraction, we find that $\psi>0.5$. If the observed S depletion in the inner solar system predated or was contemporaneous with large-scale mixing, our lower bound on the $\mathrm{S} / \mathrm{Fe}$ ratio gives an upper bound on $\psi$ of $\sim 0.65$. This measurement may be used to test mixing models of the early solar system.
\end{abstract}

Key words: comets: general - comets: individual (81P/Wild2) - Kuiper Belt - meteors, meteoroids - solar system: formation

\section{INTRODUCTION}

Here we report measurements of the chemical state of iron in cometary dust from the Jupiter-family comet Wild2, using synchrotron-based microbeam X-ray absorption spectroscopy on cometary samples returned by the Stardust mission. This comet has been in its present orbit in the inner solar system only since 1974, when it suffered a close encounter with Jupiter. Its long-term history previous to 1974 is uncertain, but it is likely an object that spent most of its life in the Kuiper Belt (Sekanina 2003), and probably formed there. Cometary dust from Wild2 was captured in low-density aerogel tiles onboard the Stardust spacecraft during a $6.1 \mathrm{~km} \mathrm{~s}^{-1}$ fly-by of the comet at $1.8 \mathrm{AU}$ in 2004 January (Brownlee et al. 2006). The sample returned to Earth in 2006 January.

\section{METHODS}

Particles captured at $\gg 1 \mathrm{~km} \mathrm{~s}^{-1}$ in aerogel form turnip- or carrot-shaped tracks, with maximum diameters and lengths of the order of 10 times and 100 times larger, respectively, than the original particle diameter (Burchell et al. 2008). Robust particles (e.g., glass or metal spheres) captured at $6 \mathrm{~km} \mathrm{~s}^{-1}$ generally survive capture without mechanical damage, but friable particles, such as the dust from the coma of Wild2, disintegrate and are later found along the entire length of the tracks, with the most massive particles forming one to several terminal tracks that extend beyond the bulb (Figure 1). Some tracks contain hundreds of particle fragments in a track bulb. Optical microscopy and micro-X-ray fluorescence ( $\mu \mathrm{XRF}$ ) mapping (see below) showed that the vast majority of particles were $\ll 10 \mu \mathrm{m}$ in size. To determine the bulk chemistry of the total iron present in a representative sample of comet Wild2 dust, we acquired $\mathrm{Fe} \mathrm{K}$-edge X-ray absorption spectra on 194 selected fragments in 11 tracks, using XRF elemental mapping of all tracks in their entirety for target identification and weighting of measurements.

\subsection{Sample Preparation}

It is generally not practical to cleanly remove all of the particles from the aerogel, so analyses were done on particles still trapped in aerogel. Using robotically controlled micromanipulators and pulled glass microneedles, we extracted 11 tracks of varying sizes and morphologies from aerogel tiles in "keystones"- tiny wedge-shaped pieces of aerogel that completely contain the impact track (Westphal et al. 2004). The keystones were typically $\sim 300 \mu \mathrm{m}$ thick and contained tracks of several hundred microns to $\sim 3 \mathrm{~mm}$ long (Figure 1). Some tracks were larger and were extracted in correspondingly larger keystones. The tracks were chosen randomly from a population at nearly normal incidence to the collector. We avoided a population of tracks that were inclined to the collector and may have resulted from a collision of a cometary particle with the central debris shield of the spacecraft (Westphal et al. 2008). The keystones were mounted either on silicon "microfork" fixtures or sandwiched between $6 \mu \mathrm{m}$ thick polypropylene films.

\subsection{XRF Mapping}

We analyzed the keystones by $\mu \mathrm{XRF}$ and micro-X-ray absorption near-edge structure ( $\mu$ XANES) spectroscopy on 


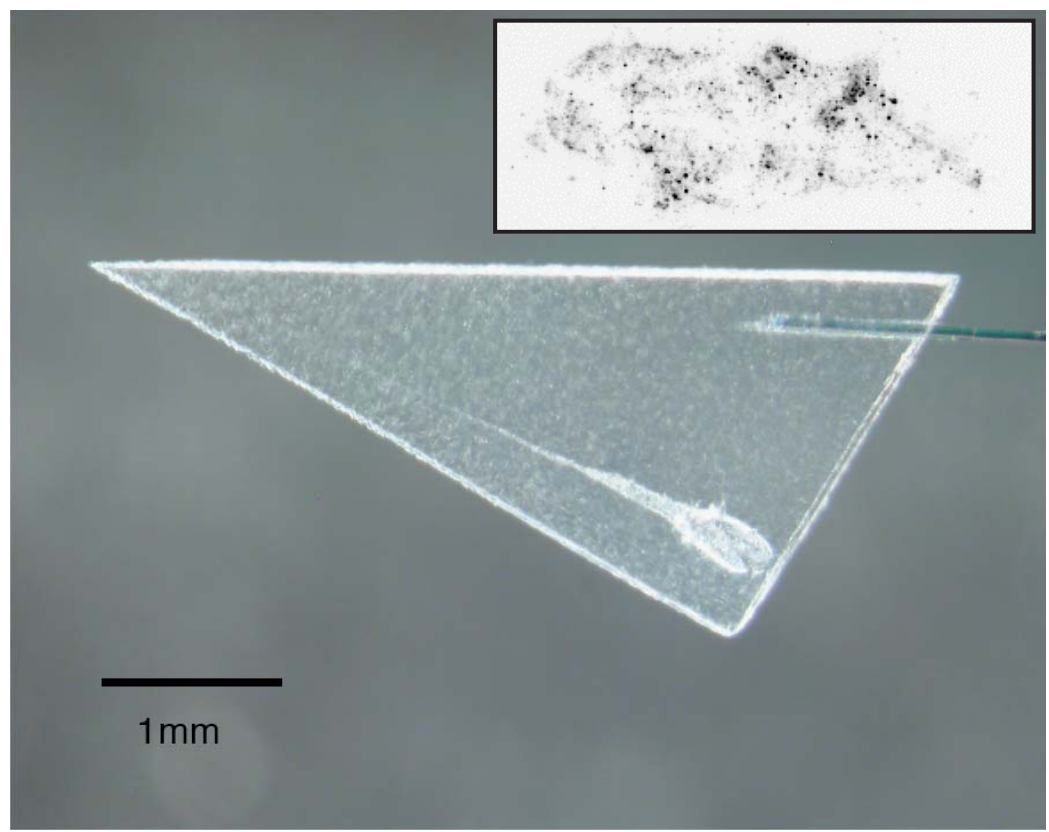

Figure 1. Track of a captured cometary dust particle (track 43) from Wild2 extracted from the Stardust collector in a thin wedge-shaped aerogel "keystone" (Westphal et al. 2004). The thickness of the keystone, in the dimension perpendicular to the page, is $300 \mu \mathrm{m}$. The fragments of the original projectile are too small to be visible in this image. The keystone is mounted on a silicon microfixture to facilitate handling. Inset: Fe $\mu$ XRF map of dissected bulb of track 74, on the same scale. The image intensity is inverted. The beam spot is $7 \mu \mathrm{m}$ in diameter.

(A color version of this figure is available in the online journal.)

beamline 10.3.2 at the Advanced Light Source, Lawrence Berkeley National Laboratory (Marcus et al. 2004). This beamline uses a $\mathrm{Si}\left(\begin{array}{lll}1 & 1 & 1\end{array}\right)$ monochromator and Kirkpatrick-Baez mirrors to focus a monochromatic ( $\sim 1 \mathrm{eV}$ width) $\mathrm{X}$-ray beam of variable spot sizes between $5 \times 5 \mu \mathrm{m}$ and $16 \times 7 \mu \mathrm{m}$. We collected maps using a seven-element Ge solid-state detector and a typical counting time of $100 \mathrm{~ms}$ per pixel. We first performed $\mu \mathrm{XRF}$ two-dimensional mapping of the major elements $(\mathrm{K}, \mathrm{Ca}$, $\mathrm{Ti}, \mathrm{Cr}, \mathrm{Mn}, \mathrm{Fe}, \mathrm{Ni}, \mathrm{Cu}$, and $\mathrm{Zn}$ ) present in the keystones using a $10 \mathrm{keV}$ incident $\mathrm{X}$-ray beam. It was not necessary to map each entire keystone: the tracks were easily visible in the beamline microscope, so only the tracks and the area immediately adjacent to them were completely mapped. We then selected Fe-rich particles in the tracks for subsequent XANES analysis at the Fe K-edge, taken to be $7110.75 \mathrm{eV}$ for Fe metal (Kraft et al. 1996).

\subsection{XANES Data Acquisition and Analysis}

For multivalent elements such as Fe the energy of the absorption edge, where the absorbance is changing very rapidly with energy, depends on the oxidation state: atoms in reduced environments start to absorb strongly several $\mathrm{eV}$ below the energy at which atoms in an oxidized environment do. The absorption edge is well determined, and alone can distinguish between oxidation states. Because of this, XANES is a particularly powerful and sensitive tool for determining oxidation state (Wilke et al. 2001; Marcus et al. 2008b). XANES spectra comparing Fe in different oxidation states are shown in Figure 2. In general, Fe $\mathrm{K}$-edge XANES spectra contain information not only on the valence but also on electronic structure (density of states) of the absorber and qualitative structural information. These spectra vary between mineral groups and between minerals within groups (O'Day et al. 2004). For example, $\mathrm{Fe}^{0}$ minerals have similar XANES spectra (Figure 3).

For most tracks, we analyzed all Fe-rich spots with photon counting rates sufficient for XANES analysis. In the case of the largest tracks, it was not practical to analyze all particles, so we measured all terminal particles and a set of randomly selected particles in the bulb. Among the 11 tracks, we collected Fe XANES spectra on 194 spots. The Fe XANES spectra were calibrated, pre-edge background subtracted and normalized using standard procedures (Kelly et al. 2008) and a suite of LabVIEW XANES programs available at ALS beamline 10.3.2 (http://xraysweb.lbl.gov/uxas/Index.htm).

\subsubsection{Fitting to Reference Spectra of Standards}

By comparing the spectrum of an unknown to a library of well characterized standards, phases can be identified, although identifications are not always unique. Identification of groups is more reliable, since many materials within a given group (e.g., sulfides) have similar XANES spectra (Figure 4). We followed two parallel, independent approaches in analyzing these data. In one approach, we fit the spectra to 56 reference spectra using the non-negative least-squares fitting function lsqnonneg in the MATLAB 7.0.4.352 (R14) library (see Table 1). In the second approach, we used least-squares fitting to choose the best fit from among $\sim 1.3 \times 10^{5}$ unique combinations of the 56 reference spectra taken three at a time. The reference spectra were acquired from 54 minerals and glasses from 24 groups (metal, sulfide, carbide, olivine, orthopyroxene, clinopyroxene, silicate glass, phyllosilicate, amphibole, sulfate, carbonate, and others; Table 1). We show typical examples of reference spectra in Figures 2 and 3. A complication is that for some non-cubic minerals polarization effects on the fine structure shape of the spectra may be important (Manceau et al. 2002), so spectra must be collected on several standard grains with different orientations. For many minerals, we collected spectra at different orientations, and used spectra from a principal components analysis as reference spectra. We categorized each standard as one of $\mathrm{Fe}^{0}$ (metals, carbides, and silicides), sulfide, or oxide (including silicates, $\mathrm{Fe}^{2+}$, or $\mathrm{Fe}^{3+}$ ), and summed the components 

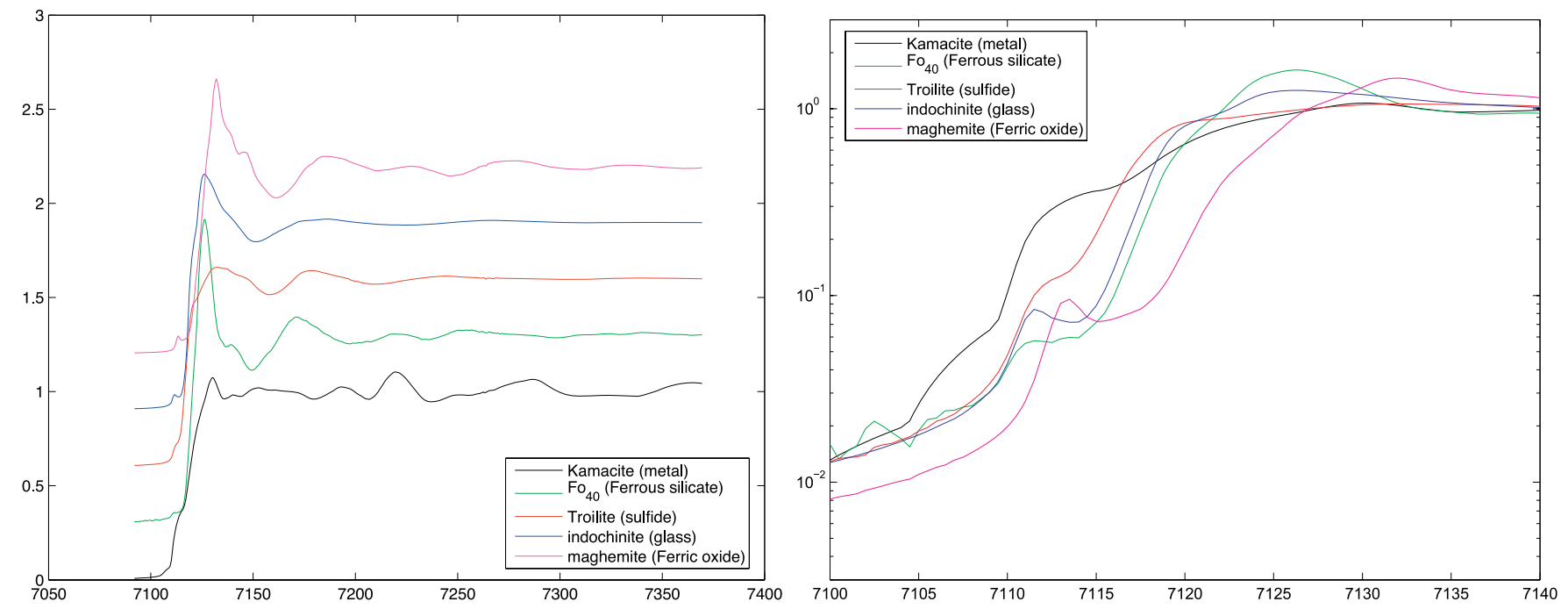

Figure 2. Left: representative Fe XANES spectra for several groups of minerals (metal, sulfide, crystalline ferric silicate, crystalline ferrous silicate, glass). Right: same as left panel, but shown as a semilog plot focussed on the edge structure between $7100 \mathrm{eV}$ and $7140 \mathrm{eV}$. The position of the edge increases systematically in energy with the oxidation state.
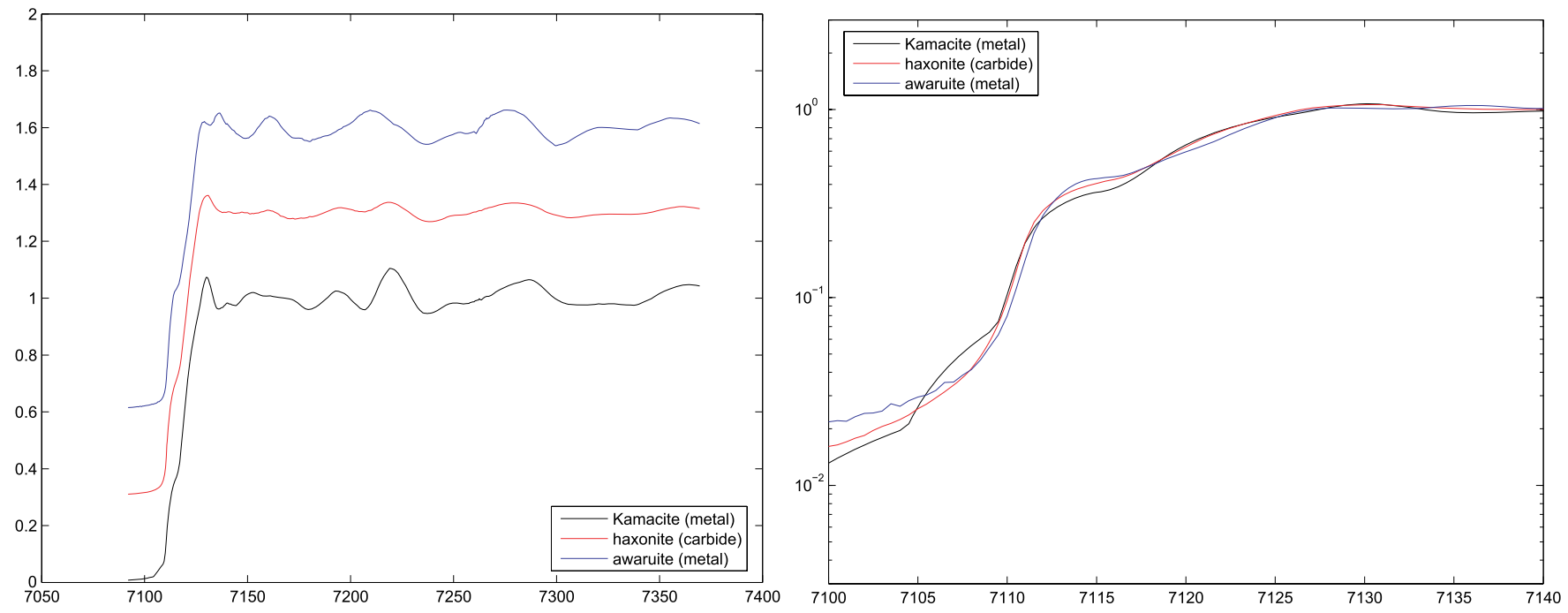

Figure 3. Representative Fe XANES spectra for $\mathrm{Fe}^{0}$ minerals (two metals and one carbide). While the edge structure is nearly identical for these three species, the post-edge oscillations are distinct.

of each fit, weighted by the magnitude of the edge jump, in each category. The magnitude of the edge jump is proportional to the concentration of $\mathrm{Fe}$ in the particle. We applied a correction factor to the particles in the bulbs of the largest tracks to account for incomplete sampling. The results of the two spectral fitting procedures were consistent.

\subsubsection{Principal Components Analysis for Multiple Polarization Components}

Since many minerals are of non-cubic symmetry, they display dichroism in the X-ray band just as they do in the visible band (Hazemann et al. 1992), and with the same second-rank tensor symmetry. Since the comet particles and, often, the particles used for our reference spectra may be single crystals, a powder-averaged spectrum is not sufficient to capture the range of signals produced by a single-mineral species. Instead, the spectrum from a grain of a given mineral will, in general, be a linear combination of a number of basis spectra which depends on the crystal symmetry. In order to produce estimates of these basis spectra, we performed XANES measurements on as many individual particles of our standards as possible. We estimated the basis spectra spanning the space of spectra from grains of arbitrary orientation. We used iterative target-factor analysis (ITFA) (Scheinost et al. 2005), an extension to principal components analysis (PCA) in which linear combinations of the PCA abstract components are formed in such a way that the resulting ITFA components are all positive, and the loadings of the individual spectra are as different as possible from each other. The number of components used is the smallest consistent with the data, and no larger than what is dictated by the crystal symmetry. We typically measured 7-9 spectra for monoclinic minerals (four independent components), and 3-4 spectra for tetragonal, hexagonal, or trigonal minerals (two independent components).

\subsubsection{Correction for Incomplete Sampling}

Due to time constraints at the synchrotron beamline, it is not practical to perform XANES analysis on every particle in a Stardust track. Following the $\mu \mathrm{XRF}$ mapping of the track, 


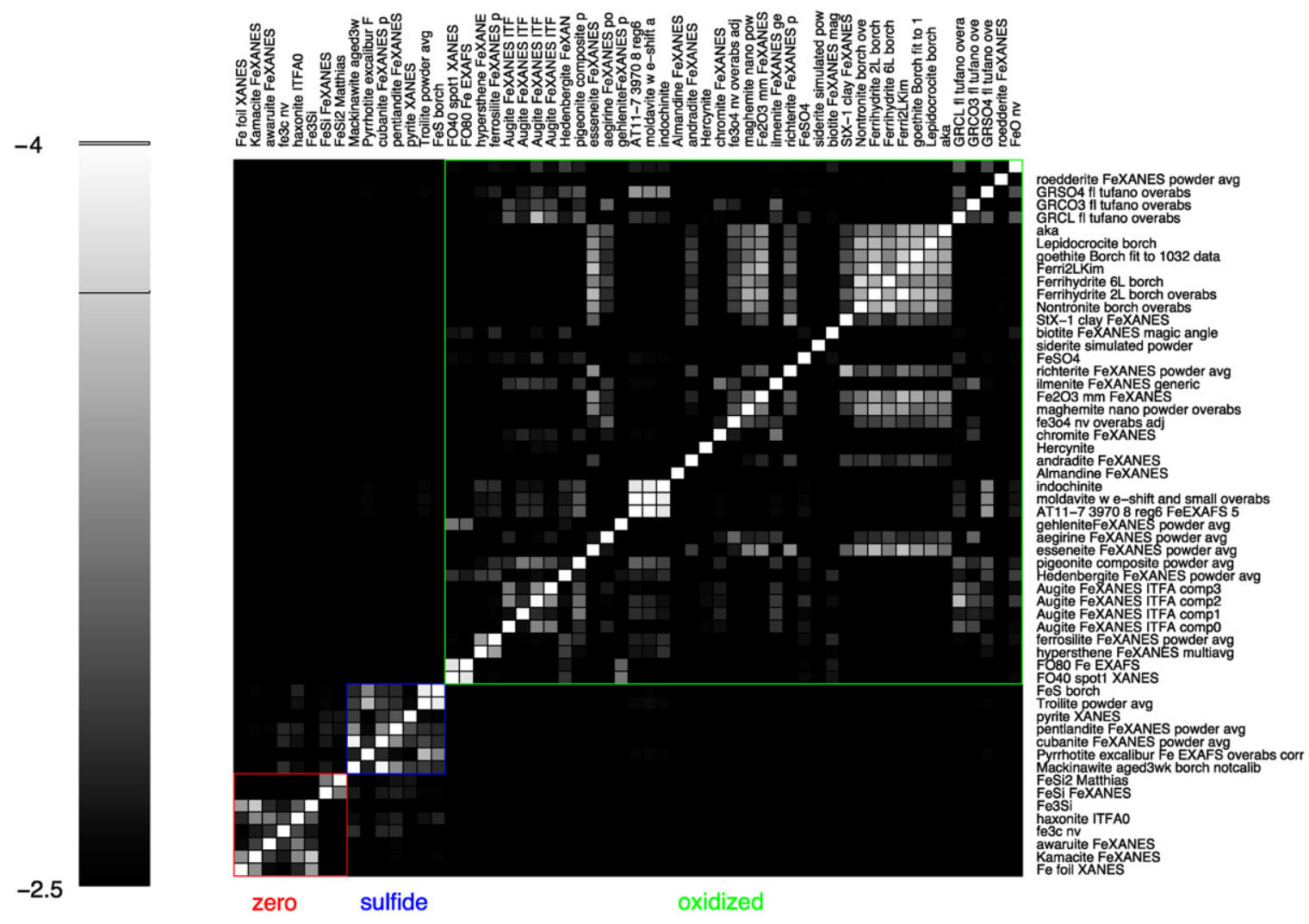

Figure 4. Degeneracy plot of the standards library. For each standard, a linear least-squares fit is performed to all the other standards in the library. The log base 10 of a mean-square residual of the fit is indicated by the color and scalebar. A good fit has a log 10 (mean-square residual) $<-3.5$. While there can be strong degeneracies within the broad groups (metals, sulfides, and oxidized phases), there is essentially no degeneracy between them. The glass standards, AT11-7, moldavite, and indochinite, are highly degenerate.

(A color version of this figure is available in the online journal.)

we chose particles with sufficient iron $\mathrm{K} \alpha$-emission signal for further XANES investigation. Consequently, more of the larger particles near the end of the track were analyzed than those in the "bulb" closer to the aerogel surface. If the composition of the comet material varies along the track, this will introduce a selection bias when calculating the bulk iron composition of the comet. In addition, the X-ray beam spot size for the XANES measurements could be smaller than the physical dimension of the particle in some cases, so less than the full XANES signal was recorded for some of the terminal particles. To account for these selection biases, we compared the integrated fluorescence intensity from the backgroundsubtracted XRF maps to the summed XANES edge jumps to compute undersampling correction factors for the bulbs and terminal particles of each track. This correction factor was applied to the weightings of the XANES measurements.

\subsubsection{Verification of Accuracy and Sensitivity of Fitting Procedure}

We evaluated the accuracy of the fitting procedure by fitting single standards and combinations of standards against the standard library with those standards removed, and confirming that the correct fractions were calculated. We also tested the linearity and sensitivity of the fitting procedure by mixing spectra of representative standards (metals, sulfides, $\mathrm{Fe}^{2+}$-bearing minerals, and $\mathrm{Fe}^{3+}$-bearing minerals) into the Wild2 spectra at several levels between 0 and $100 \%$, and confirming that the mixture was reflected accurately in the calculated ratios. We found typical errors induced by cross-family degeneracy in our measurement of the Wild 2 compositions in the $\mathrm{Fe}^{0}$, sulfide, and oxidized $\mathrm{Fe}$ fractions of approximately 5\%. The maximum deviation in $\xi$ from the true value, $15 \%$, corresponded to a mixture of equal amounts of sulfide and $\mathrm{Fe}^{3+}$. This deviation is unlikely because this composition is far from that of Wild2. This systematic error is small compared to the statistical errors in all of our measurements. Although we have included all of the fits in our reported value, we found that $\xi$ is insensitive to the position of a cut in the residual.

\subsubsection{Correction for Overabsorption}

Saturation (overabsorption) effects can occur in XANES measurements performed in fluorescence mode (Manceau et al. 2002). If the particle has a thickness comparable to the absorption depth of the incident radiation, then the varying penetration depth as a function of energy will cause a distortion of the spectrum. For four of the 194 spectra collected on Fe hot-spots, linear combination fitting did not provide conclusive results and overabsorption effects were suspected based on the comparison of these spectra with the standards. Those spectra were fitted with a correction for overabsorption using a simple model (Manceau et al. 2002). These spots together comprised $\sim 10 \%$ of the $\mathrm{Fe}$ used in the entire analysis. The accuracy of the fitting after the absorption correction is expected to be better than $10 \%$, so the overall systematic error is $\sim 1 \%$. We conclude that overabsorption is not a significant source of systematic error.

\subsubsection{Summary of Track Analysis}

In Table 2, we present a summary of the results of our analysis. The entire data set is available in the supporting materials online. 

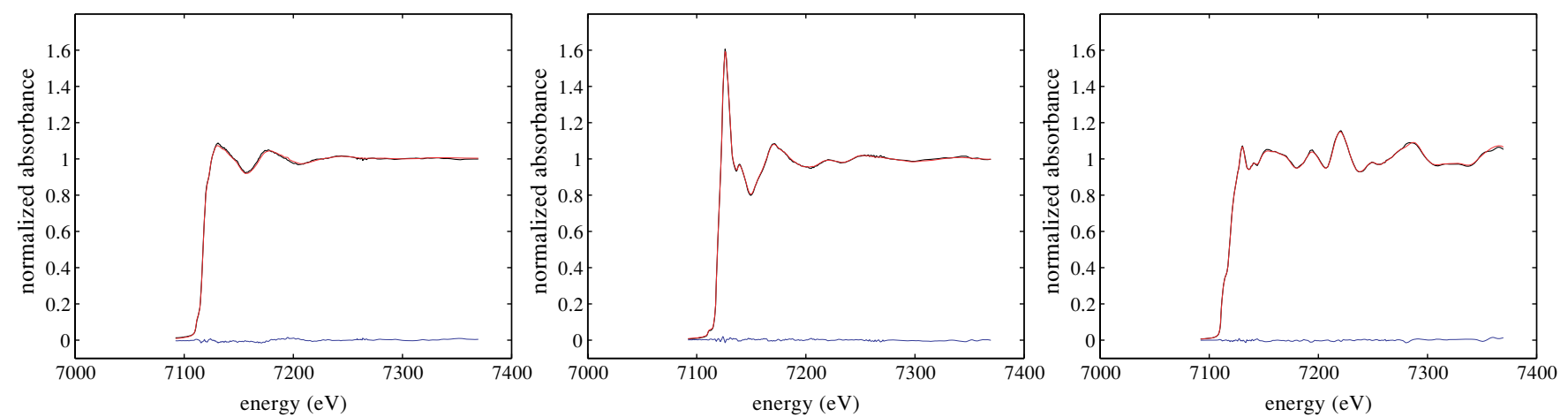

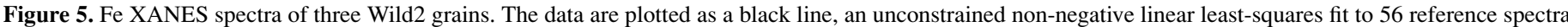

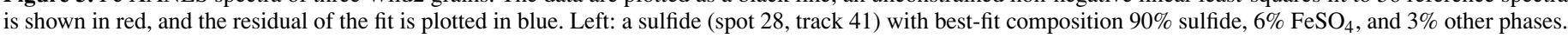

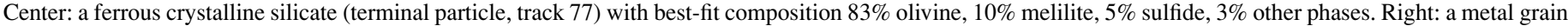
(spot 2, track 38) with best-fit composition $90 \%$ Fe metal, $7 \%$ awaruite, and $3 \%$ other phases.

(A color version of this figure is available in the online journal.)

Table 1

Standards Used in the Analysis. The Library of Standards is Available in the Online Journal

\begin{tabular}{|c|c|}
\hline \multicolumn{2}{|c|}{$\mathrm{Fe}^{0}$} \\
\hline Fe Foil & Kamacite \\
\hline Awaruite & $\mathrm{Fe}_{3} \mathrm{C}$ \\
\hline Haxonite & $\mathrm{Fe}_{3} \mathrm{Si}$ \\
\hline $\mathrm{FeSi}$ & $\mathrm{FeSi}_{2}$ \\
\hline \multicolumn{2}{|c|}{ Sulfides } \\
\hline Mackinawite & Pyrrhotite \\
\hline Cubanite & Pentlandite \\
\hline Pyrite & Troilite \\
\hline Synthetic FeS & \\
\hline \multicolumn{2}{|c|}{ Oxidized Fe } \\
\hline Forsterite $_{40}$ & Forsterite $_{80}$ \\
\hline Hypersthene & Ferrosilite \\
\hline Augite & Hedenbergite \\
\hline Pigeonite & Esseneite \\
\hline Aegirine & Gehlenite \\
\hline Undersea Basalt Glass & Moldavite Glass \\
\hline Indochinite Glass & Almandine \\
\hline Andradite & Hercynite \\
\hline Chromite & $\mathrm{Fe}_{3} \mathrm{O}_{4}$ \\
\hline Maghemite & Magnetite \\
\hline Ilmenite & Richterite \\
\hline $\mathrm{FeSO}_{4}$ & Siderite \\
\hline Biotite & StX-1 Clay \\
\hline Nontronite & Ferrihydrite (3) \\
\hline Goethite & Lepidocrocite \\
\hline Akagenite & Chloride Green Rust \\
\hline Carbonate Green Rust & Sulfide Green Rust \\
\hline Roedderite & $\mathrm{FeO}$ \\
\hline
\end{tabular}

\subsubsection{Determination of Confidence Limits}

We used a Monte Carlo based approach to determine statistical confidence limits in the metal+sulfide-to-oxide ratio $\xi$ (Chernick 1999). This approach is equivalent to asking the following question: if this experiment were performed again, choosing a different set of 11 tracks at random from the ensemble of all tracks, how different would our result be? We assumed, because we must, that the diversity among the 11 tracks themselves is representative of that of the entire collection. In each Monte Carlo trial, we chose 11 tracks randomly, one at a time, from among the 11 tracks in our data set. We assembled a new data set from this set of 11 tracks, and computed $\xi$. The entire calculation comprised an ensemble of 10,000 trials. We then sorted the calculated ratios $\xi$, and chose the $228^{\text {th }}$ and $9772^{\text {nd }}$ entries in the sorted list as the $2 \sigma$ lower and upper limits, respectively. We also used the same method to compute confidence limits using only the set of 194 particles, without regard to track assignments. However, this resulted in narrower confidence limits. This is evidence that the compositions within tracks were strongly correlated with each other. We therefore chose to use the more conservative track-based, rather than the particle-based, calculation. This method was also used by Flynn et al. (2006).

In the ternary diagram $\left(\mathrm{Fe}^{0}\right.$ versus oxide versus sulfide), we computed confidence limits using a similar Monte Carlo method. Each Monte Carlo trial resulted in a single point on the ternary diagram. In a procedure similar to that of the computation of one-dimensional confidence limits, we computed the median value of the distribution, then determined the diameter of the circle on the plot that included $95.4 \%$ of the points. The allowed region is then determined by the envelope of points that fall within this circle.

\section{RESULTS}

Using the procedure described in Section 2, we computed the molar ratio $\xi$ of reduced iron (metals, carbides, silicides, and sulfides) to oxidized $\mathrm{Fe}$. We found that $\xi=2.34_{-1.56}^{+1.77}$ (stat., $2 \sigma$ ). Because the cometary particles were captured in silica aerogel, we could not measure their Si content. Thus, our measurement appears as a band in a Si-normalized plot of $\mathrm{Fe}^{0}+$ sulfide versus oxidized Fe (Figure 6). We also computed the allowed $2 \sigma$ region in the ternary $\mathrm{Fe}^{0}$ versus oxide versus sulfide diagram (Figure 7). Here carbides and silicides are classified as $\mathrm{Fe}^{0}$ along with metals (Marcus et al. 2008b).

We also measured the relative amorphous and crystalline fractions of Fe-bearing silicates. We found that the crystalline fraction $\eta$ is $0.59_{-0.10}^{+0.37}$ (two-sided, $2 \sigma$ ), and is $>0.50$ (one-sided lower limit, $2 \sigma$ ).

The effects of hypervelocity capture on the oxidation state of cometary material are a significant concern. The timescale for particle capture is tens of nanoseconds. Transmission electron micrograph (TEM) observations have shown that particle surfaces are strongly heated, but below a micron from their surfaces they are well preserved. Using Fe $\mu$ XANES and TEM, we have observed systematic reduction of Fe during hypervelocity capture of basalt glass projectiles into aerogel at $6 \mathrm{~km} \mathrm{~s}^{-1}$ (Marcus et al. 2008a). This reduction results in iron carbide $\left(\mathrm{Fe}_{3} \mathrm{C}\right)$, not 


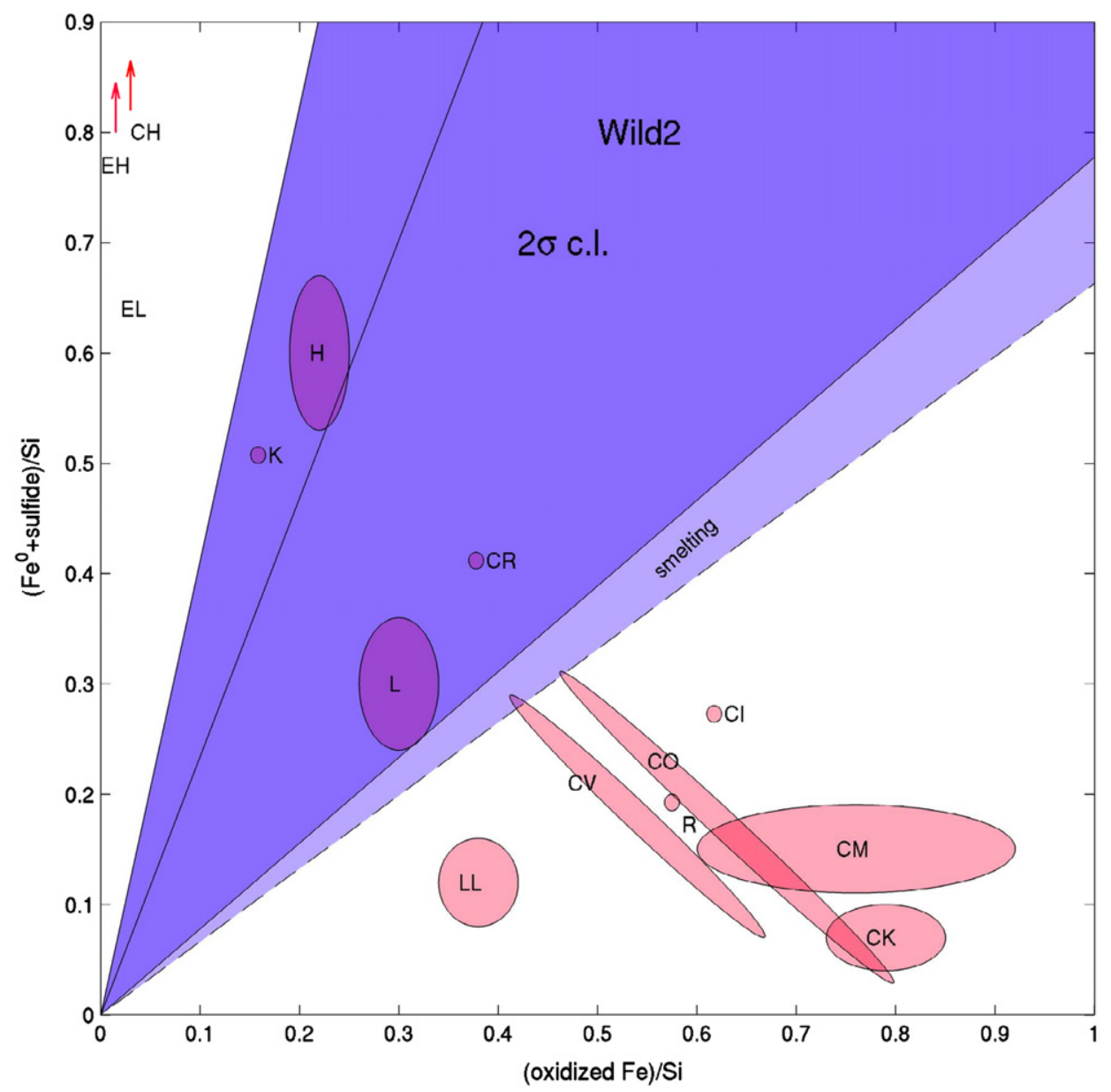

Figure 6. Urey-Craig plot of $\mathrm{Fe}$ in $\mathrm{Fe}^{0}+$ sulfides vs. oxidized $\left(\mathrm{Fe}^{2+}\right.$ and $\left.\mathrm{Fe}^{3+}\right)$, normalized to Si, for carbonaceous (CI, CM, CO, ...), ordinary (H, L, LL) and enstatite $(\mathrm{EH}, \mathrm{EL})$ chondrite groups and comet Wild2. Red regions representing meteorite groups encompass the observed dispersions within the groups, with the exception of $\mathrm{EH}, \mathrm{K}, \mathrm{CR}$, and $\mathrm{CI}$ which are based on single measurements. The dark blue region is the two-dimensional $2 \sigma$ allowed region in the mean values for the ensemble of 11 tracks that we analyzed. The dashed region shows the effect of assuming that all carbide components originated as oxides and were reduced to iron carbide. Data from meteorite groups are given by Jarosewich (1990), Yanai (1992), and Mason \& Wiik (1966, 1962b, 1962a).

(A color version of this figure is available in the online journal.)

Table 2

Summary of Track Composition

\begin{tabular}{lcccccc}
\hline \hline Track & Fragments Analyzed & ${\text { Fraction } \mathrm{Fe}^{0}}$ & Fraction Sulfide & Fraction Oxidized & Crystalline Silicate Fraction & Total Relative Weight \\
\hline 38 & 3 & 0.17 & 0.05 & 0.78 & 1.00 & $3.4 \times 10^{-2}$ \\
41 & 33 & 0.16 & 0.52 & 0.32 & 0.46 & 1.00 \\
42 & 3 & 0.00 & 0.96 & 0.04 & 0.77 & $4.1 \times 10^{-5}$ \\
43 & 4 & 0.10 & 0.29 & 0.61 & 1.00 & $4.7 \times 10^{-2}$ \\
44 & 1 & 0.41 & 0.10 & 0.49 & 1.00 & $6.8 \times 10^{-4}$ \\
54 & 2 & 0.08 & 0.09 & 0.83 & 0.52 & $5.0 \times 10^{-4}$ \\
74 & 56 & 0.06 & 0.38 & 0.56 & 0.99 & $2.9 \times 10^{-1}$ \\
77 & 53 & 0.13 & 0.36 & 0.51 & 0.96 & $8.2 \times 10^{-2}$ \\
103 & 2 & 0.51 & 0.28 & 0.20 & 0.82 & $1.6 \times 10^{-5}$ \\
104 & 6 & 0.04 & 0.28 & 0.68 & 0.71 & $2.2 \times 10^{-4}$ \\
105 & 31 & 0.08 & 0.60 & 0.32 & $4.3 \times 10^{-2}$ \\
\hline
\end{tabular}

(Data for all 194 points are available in supplemental data files (tar.gz) in the online journal. A portion is shown here for guidance regarding its form and content)

iron metal, and is reminiscent of smelting - that is, the reduction of Fe oxide by reaction with carbon. Carbide is $\sim 15 \%$ of the $\mathrm{Fe}^{0}$ in our fits. We conclude that melting and reduction are unlikely to have played a major role in the capture of Stardust samples, but are nevertheless the sources of systematic uncertainty, and lower the $2 \sigma$ lower limit on $\xi$ from 0.78 to 0.67 .
Our results including this systematic error are shown as the dashed regions in Figures 6 and 7. Sulfides can be converted to metal through loss of $\mathrm{S}$ by heating. Loss of sulfur from $\mathrm{Fe}-\mathrm{Ni}$ sulfides attributed to capture effects in Stardust aerogel was reported by Zolensky et al. (2006). Some captured sulfide grains showed both Fe metal and troilite present. Our Fe XANES 


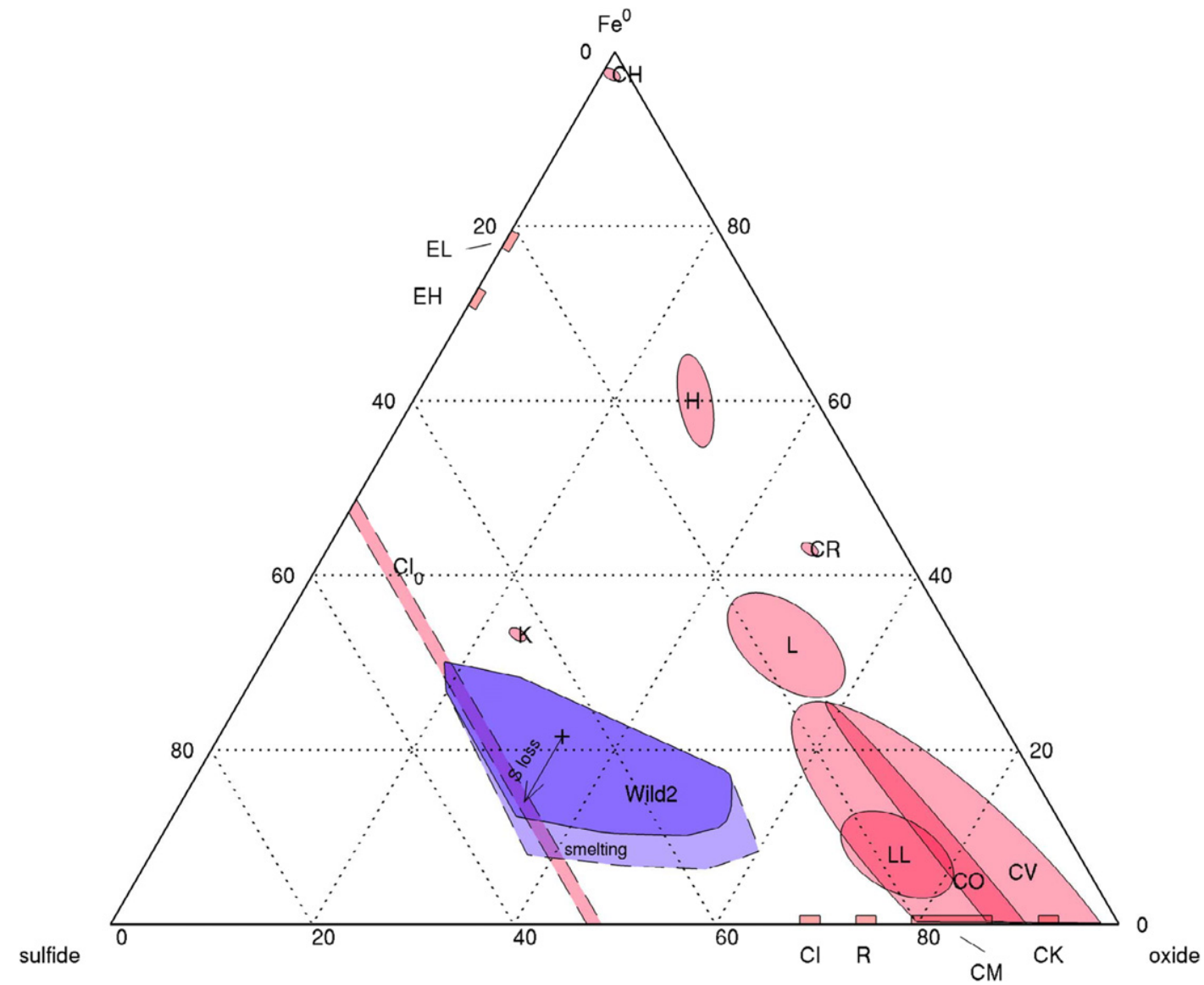

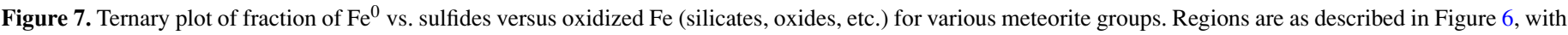

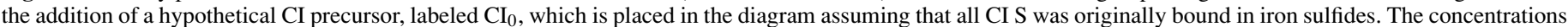

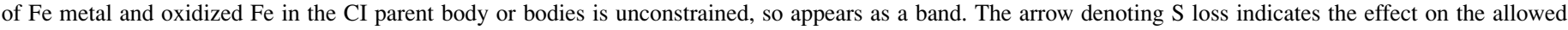
confidence region of sulfide loss during capture in aerogel.

(A color version of this figure is available in the online journal.)

measurements distinguish between iron metal and FeS resulting in an accurate sulfide count for the returned samples. Because of their low melting point, sulfides may also melt and be preferentially widely dispersed in the tracks. Sulfur loss from sulfides during capture decreases the overall iron sulfide/metal, meaning the comet dust was likely to be more sulfide-rich than measured. The arrow in Figure 7 shows the effect from sulfide reduction.

Hypervelocity capture may also convert crystalline silicates into amorphous phases. The reverse process is unexpected because of the extremely short capture timescales. This expectation is born out in the XANES measurements on the basalt shot discussed above-here we saw no evidence of crystalline silicates in the tracks. We are also unaware of any reports of crystalline silicate production in the literature on hypervelocity capture in aerogel. We thus treat the measurement of the crystalline silicate fraction as a lower limit on the actual abundance in the comet.

Flynn et al. (2006) reported synchrotron X-ray fluorescence measurements of abundances of elements from $S$ through Se. They reported that $S$ was significantly underabundant $(\mathrm{S} / \mathrm{Fe}$ $\sim 0.2 \times \mathrm{CI})$, but that the moderately volatile chalcophile elements $\mathrm{Cu}, \mathrm{Zn}$, and Ga were significantly $(\sim 2 \sigma)$ overabundant as compared with CI meteorites, which are thought to represent the bulk solar-system abundances. They pointed out a strong possibility that $S$ was underestimated in the fluorescence measurements because of attenuation of $\mathrm{S} \mathrm{K} \alpha \mathrm{X}$-rays in the sample. The absorption length of $\mathrm{S} K \alpha \mathrm{X}$-rays in iron sulfides is $\sim 2 \mu \mathrm{m}$. Our measurement of the abundance of FeS with respect to total $\mathrm{Fe}$ does not suffer a problem of $\mathrm{S} \mathrm{K} \alpha$ X-ray attenuation in large particles, because we use $\mathrm{Fe} \mathrm{K} \alpha \mathrm{X}$-rays in our measurements, for which absorption in the sample is negligible. We find that the $S_{\text {sulfide }} / F e$ ratio $\zeta=0.46_{-0.19}^{+0.05}(2 \sigma$, twosided confidence limit (c.l.)) (Figure 7) or alternatively $\zeta>0.31$ ( $2 \sigma$, one-sided c.1.). This ratio normalized to CI (Lodders 2003) is $\mathrm{S}_{\text {sulfide }} / \mathrm{Fe}=0.89_{-0.37}^{+0.10} \times \mathrm{CI}$, or $\zeta>0.60 \times \mathrm{CI}(2 \sigma$, one-sided c.l.). Our value may be considered to be a lower limit on the overall sulfur abundance, since our measurement is blind to $S$ in non-Fe-bearing phases and $\mathrm{S}$ volatilized during capture into aerogel. Zolensky et al. (2006) estimated that around half of the sulfide grains analyzed during preliminary examination showed evidence of sulfur loss, often increasing metallic Fe in particles. By estimating the amount of sulfur lost from a collection of Wild2 iron sulfide grains using the Zolensky et al. data, we find that our lower bound increases to $\sim 0.8$. We conclude that sulfur is not significantly depleted with respect to $\mathrm{CI}$ and is consistent with the abundance of volatile chalcophile elements observed in Wild2.

\section{COMPARISON OF P81/WILD2 WITH THE CHONDRITIC METEORITE COLLECTION}

Urey \& Craig (1953) were the first to review the systematic variation of Fe-oxidation state between meteorite groups. In 


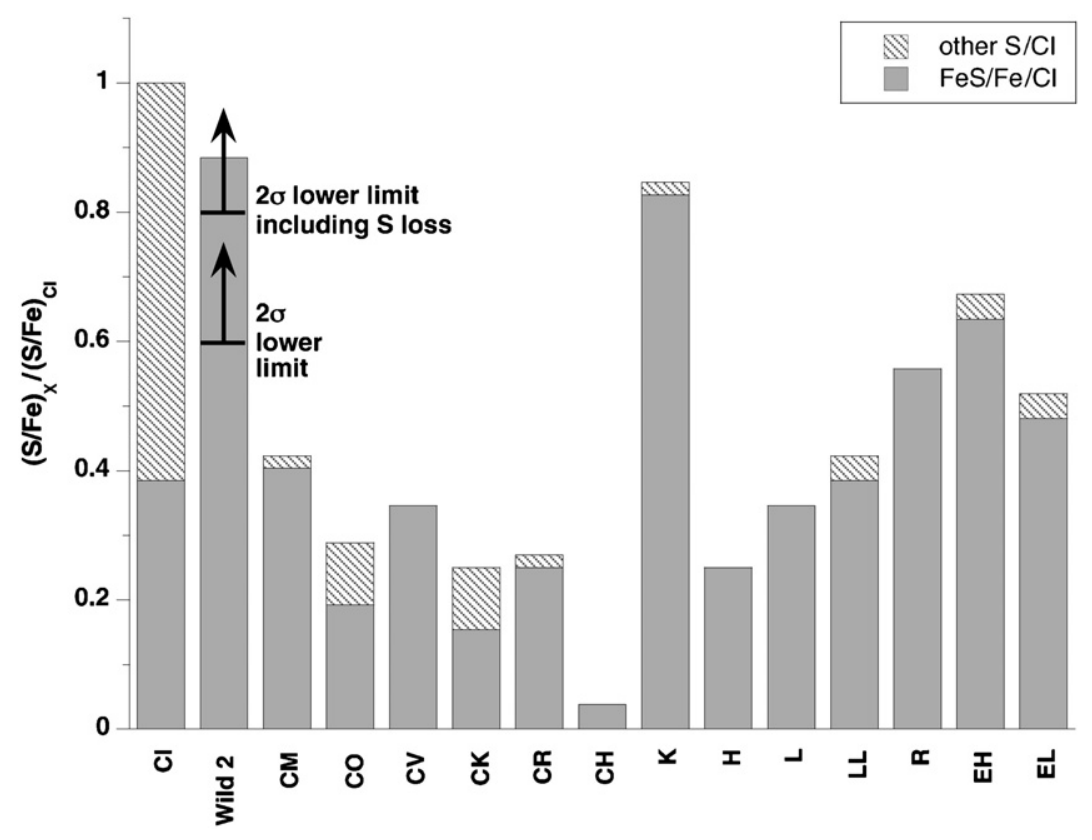

Figure 8. Bulk S/Fe for Wild2 and meteorite groups. Data from Tables 16.10 and 16.11 of Lodders \& Fegley (1998) with additional CI data from Bland et al. (2004). The measurement for $81 \mathrm{P} / \mathrm{Wild} 2(\mathrm{~S} / \mathrm{Fe}=0.89 \times \mathrm{CI}$, median value) includes only the sulfur in iron sulfides (solid gray), and does not include sulfur in other non Fe-bearing phases (hashed). The calculated $2 \sigma$, one-sided S/Fe lower limit is $0.6 \times \mathrm{CI}$ (lower arrow). If we account for sulfur volatilized during capture (see text), the lower limit increases to $0.8 \times \mathrm{CI}$ (upper arrow).

the Urey-Craig plot of concentration of metal + sulfides versus concentration of oxidized iron $\left(\mathrm{Fe}^{2+}\right.$ and $\left.\mathrm{Fe}^{3+}\right)$, both normalized to $\mathrm{Si}$, meteorite groups fall into distinct, mostly non-overlapping regions (Figure 7). The carbonaceous chondrites $(\mathrm{CR}, \mathrm{CO}, \mathrm{CV}$, $\mathrm{CM}, \mathrm{CI}, \mathrm{CK}$ ) are oxidized to various degrees, with the CI group extensively oxidized by aqueous alteration. The enstatite chondrite groups EH and EL contain essentially no oxidized Fe. The ordinary chondrites and the grouplet K (Kakangari) are intermediate in the oxidation state.

We compare our measurements to the bulk oxidation sulfidization state of $\mathrm{Fe}$ in the recognized families of carbonaceous chondrites. This comparison is only meaningful to the extent that systematic selection effects in the dust release and acceleration process are not important, and that the Stardust cometary sample is representative of the bulk comet. We cannot exclude the possibility that global redistribution of material occurred during the evolution of the comet, but this seems unlikely given its cryogenic state. The allowed region includes the ordinary chondrite group $\mathrm{H}$ and the unclassified group $\mathrm{K}$, and is marginally consistent $(\sim 2 \sigma)$ with the ordinary group $\mathrm{L}$ and the carbonaceous groups CR. On the assumption that all of the carbide components are the result of smelting of oxides during collection, the results are marginally consistent with the $\mathrm{CV}$ and $\mathrm{CO}$ groups. However, the relative fractions of metal and sulfide (Figure 7) in Wild2 are inconsistent at the $>2 \sigma$ level with any recognized meteorite group. We note that any conversion of sulfides to metals during hypervelocity capture in aerogel would imply that Wild 2 is even more sulfide-rich than our measured value (Figure 8), which is already more sulfide-rich than any other meteorite group except $\mathrm{K}$.

Although the Fe-oxidation state clearly has fundamental significance, a definitive physical mechanism or set of mechanisms for defining oxidation state remains elusive. The meteorite collection spans an enormous range of oxidation states, from orders of magnitude more reduced ( $\mathrm{E}$ chondrites) to orders of magnitude more oxidized (CI chondrites) than a cooling gas of solar composition (Krot et al. 2000). From thermodynamic considerations, Wasson (1985) and Rubin \& Wasson (1995) have suggested that the oxidation state is a reflection of heliocentric distance at the time of meteorite parentbody formation, with oxidation increasing with heliocentric distance. That the terrestrial planets apparently condensed from reduced material (Javoy 1995) and the Jovian planets are highly water-rich (Krot et al. 2000) supports this idea. The oxidized carbonaceous chondrites would have formed at the greatest heliocentric distance, and the reduced E chondrites at the smallest, with the ordinary chondrites intermediate. If this were to hold uniformly, comets which formed far from the Sun would then be expected to be highly oxidized like the carbonaceous chondrites. Our observation that Wild2 is reduced compared to the carbonaceous chondrites at the very least implies that the situation must have been considerably more complex.

$81 \mathrm{P} / \mathrm{Wild} 2$ is similar in its average oxidation state to ordinary chondrites (Figure 6). The ordinary chondrites are the most common class of meteorite, and consist of three groups, H, L, and LL, which are distinguishable by their bulk composition, mineralogy, and oxygen isotopic composition. Although ordinary chondrites are depleted in volatile siderophile and chalcophile elements such as Ga, Sb, Se, and Zn (Brearley \& Jones 1998) compared to CI meteorites, the concentrations of the volatile lithophile elements (e.g., Na, K) are nearly identical to CI. Thus they cannot be considered volatile-poor. Some of the most primitive carbonaceous chondrites, such as the CO3.0 chondrites and the least aqueously altered of the CR chondrites, as well as a class of ordinary chondrites, the unequilibrated ordinary chondrites, have a wide range of fayalitic olivine content. Zolensky et al. (2006) reported a wide range of olivine compositions in Wild2 ( $\left.\mathrm{Fa}_{\sim 0-96}\right)$, which is similar to the distribution in some unequilibrated ordinary chondrites, e.g., the meteorite Semarkona. Only the CI group has a higher bulk S/Fe content than Wild 2 (Figure 8), though CI is more oxidized than Wild 2 (Figure 6) due to extensive aqueous alteration on the parent body. Before alteration, the sulfur in CI was originally present as troilite 
(Bullock et al. 2005), and only this hypothetical unaltered CI, as shown in Figure 7, could have a similar sulfide content and iron chemistry as Wild2.

Based on the lack of glass embedded with metals and sulfides (GEMS), the crystallography of an enstatite whisker, and other lines of evidence, Ishii et al. (2008) have recently reported that returned Stardust samples from Wild2 are not similar to chondritic-porous interplanetary dust particles (IDPs), but instead more closely resemble asteroidal material, as represented in collections of chondritic meteorites. Our measurement points away from this conclusion. Apparently, the response to the question regarding the Stardust cometary sample that was posed before its return- "Have we seen this material before?" (Westphal et al. 2004)—is "no."

\section{ESTIMATE OF THE MIXING FRACTION OF INNER NEBULAR MATERIAL}

The presence of crystalline silicates in the comae of comets, inferred through infrared observations (Hanner 1994), has been a long-standing puzzle. Crystalline silicates are unexpected in comets if comets are composed of pristine interstellar material (Greenberg 1998), since interstellar silicates are almost entirely amorphous (Kemper et al. 2004). Heating to high temperatures $(>1100 \mathrm{~K})$ in principle can anneal amorphous silicates to crystallinity, but potential heating sources in the protoplanetary disk (nebular shocks (Harker \& Desch 2002), steady infall of gas from the parent cold molecular cloud (CMC) (Ruzmaikina \& Ip 1994), and cloudlet accretion (Boss \& Graham 1993; Tanaka et al. 1998)) do not appear to have been sufficiently strong to heat materials in the outer nebula to such high temperatures.

This conundrum led to the suggestion by several groups that large-scale mixing may have been important in the protoplanetary disk (Nuth \& Johnson 2006; Shu et al. 1996; Ciesla 2007; Prinn 1990). Recent reports of highly refractory calciumaluminium rich inclusions (CAI)-like objects (Brownlee et al. 2006) and large concentrations of noble gases (Marty et al. 2008) in Stardust samples underscore the need for such mixing. Two general classes of mechanisms have been proposed. Partially inspired by astronomical observations of bipolar outflows in protoplanetary accretion disks, Shu and colleagues (Shu et al. 1996) proposed that disk material may have been entrained in a magnetohydrodynamically driven outflow, originating at the inner edge of the accretion disk at $\sim 0.06$ AU. Material from this outflow would have rained down on the entire protoplanetary disk, possibly enriching it in highly refractory materials. The other possibility is that large-scale turbulent mixing occurred in the disk (Bockelée-Morvan et al. 2002; Ciesla 2007). Onedimensional turbulent models suffered from the difficulty that diffusion over very large distances was expected to be inefficient because it competed with a large-scale sunward accretionary flow (see, however, Prinn 1990).

Based on these observations and on the existence of plausible mixing models, there is now general consensus that inner solar system material is present in comets. However, the evidence from the Stardust samples until now has been largely anecdotal, and it has not been possible to place quantitative constraints on the mixing fraction.

To be definite, we consider the following two-component mixing model. We assume that the comet is composed of a fraction $\psi$ of inner solar-system material, and a fraction $1-\psi$ of unaltered material inherited from the CMC. These two reservoirs had distinct crystalline silicate fractions and $\mathrm{S} / \mathrm{Fe}$ ratios. The silicates in the CMC material were interstellar and so essentially entirely amorphous (Kemper et al. 2004). The sulfur was mostly contained in FeS (Keller et al. 2006), although small amounts of volatile sulfur compounds $\left(\mathrm{H}_{2} \mathrm{~S}\right.$, OCS, $\mathrm{SO}$, and others) would have been present (Palumbo et al. 1997). $\mathrm{H}_{2} \mathrm{~S}$ is commonly observed in cometary comae (Irvine et al. 2000). We assume that the solar system inherited the $\mathrm{S} / \mathrm{Fe}$ ratio of the CMC, so the $\mathrm{CMC}$ elemental $\mathrm{S} / \mathrm{Fe}$ ratio was solar.

In considering the inner solar-system reservoir, by "inner solar-system" we mean that part of the solar system in which conditions are favorable for producing crystalline silicates. This could either be a hot region at early times at heliocentric distance $<4$ AU with a temperature $>1100 \mathrm{~K}$ (Hersant 1994), or it could be a cooler region at later times at 2-10 AU in which interplanetary shocks periodically heat and vaporize dust, and also produce chondrules (Harker \& Desch 2002). In this environment, we conservatively assume that all silicates rapidly become crystalline. (We discuss this assumption further below.) We have abundant samples of inner solar-system material in the form of the chondritic meteorites; they are generally depleted by factors of 3-5 with respect to $\mathrm{CI}$ in the moderately volatile elements (MOVEs), including $\mathrm{S}$. (The major exceptions are CI, $\mathrm{EH}$, and $\mathrm{K}$; CI may have formed at a large heliocentric distance and so escaped the $\mathrm{S}$ depletion (Gounelle et al. 2006); the origin of CI-like $\mathrm{S}$ in $\mathrm{EH}$ chondrites is not clear; $\mathrm{K}$ (Kakangari) is enigmatic, rare, and may be pathological (Davis et al. 1977).) Further, the Earth appears to be S-poor, with an $\mathrm{S} / \mathrm{Fe}$ ratio $\sim 0.05 \times$ CI (Allègre et al. 2001).

The physical origin of the MOVE depletion is uncertain. The two prominent competing models are the incomplete condensation model (Wasson \& Chou 1974; Bland et al. 2004) and a two-component mixing model (Alexander et al. 2001). Recently, Yin has suggested that the S depletion was inherited from incomplete condensation in the interstellar medium (Yin 2005), although evidence from $\mathrm{Rb} / \mathrm{Sr}$ isotopes poses challenges to this model (Palme 2001). The timing of this $\mathrm{S}$ depletion is not well constrained, except that it must have predated chondrule formation. Nevertheless, regardless of the mechanism and timing, the depletion in $\mathrm{S}$ in inner solar-system material appears to be an observational fact.

We now consider constraints that our measurements impose on the inner nebular mixing fraction $\psi$. First, we consider the crystalline silicate fraction. Our analytical technique is only sensitive to Fe-bearing silicates. The fraction of $\mathrm{Fe}$ atoms residing in silicates in the inner nebula and CMC are $x_{\text {in }}$ and $x_{\mathrm{cmc}}$, respectively. Then the crystalline fraction $\eta$ is given by

$$
\eta=\frac{(1-\psi) x_{C M C} \eta_{C M C}+\psi x_{\mathrm{in}} \eta_{\mathrm{in}}}{(1-\psi) x_{C M C}+\psi x_{\mathrm{in}}}
$$

where we assume that $\eta_{C M C}=0$ and $\eta_{\text {in }}=1$. Defining $f \equiv x_{\mathrm{cmc}} / x_{\mathrm{in}}$, the inner nebula fraction $\psi$ is related to $\eta$ by

$$
\psi=\frac{f}{1 / \eta-1+f} .
$$

We consider only the lower limit on the measured crystalline fraction $\eta$ because crystalline silicates may be destroyed during capture, and because melted and compressed aerogel with dissolved Fe may be indistinguishable from any possible cometary amorphous silicates. Our lower limit on the crystalline silicate fraction is $\eta>0.50$ (one-sided lower limit, $2 \sigma$ ). If we assume that the average fraction of $\mathrm{Fe}$ atoms in silicates in the inner nebula and the CMC are the same, that is, $f=1$, this gives

$$
\psi>0.50
$$


This result depends on the assumption that the formation of the majority of crystalline silicates predated or was concurrent with strong nebular mixing. Scott \& Krot (2005) have reported that the matrices of primitive chondrites contain abundant amorphous ferroan silicates, and have suggested that these are formed by irradiation or vaporization and rapid cooling, probably in shocks. The presence of amorphous ferroan silicates in the inner solar system (i.e., $\eta_{\text {in }}<1$ ) would require stronger mixing, i.e., a larger value of $\psi$, still consistent with our lower limit.

Next we consider the constraint on $\psi$ imposed by the measured elemental $\mathrm{S} / \mathrm{Fe}$ ratio $\zeta$. We found that $\zeta>0.31$ ( $2 \sigma$, one-sided c.l.). We use the lower limit here because of the possibility of $S$ loss during capture, and the possibility of $\mathrm{S}$ residing in non-Fe-bearing phases to which we are not sensitive. From the values for the chondritic meteorites, we take the average atomic $\mathrm{S} / \mathrm{Fe}$ ratio $s_{\text {in }}^{\prime}$ in inner solar-system material to be 0.18 (Figure 8 ), or approximately $0.35 \times$ CI. We define $g=y_{\mathrm{cmc}} / y_{\text {in }}$, where $y_{\text {in }}$ and $y_{\mathrm{cmc}}$ are the molar fraction of $\mathrm{Fe}$ atoms in material in the CMC and inner nebula, respectively. It is not clear how to calculate this precisely from the meteorite groups, since the weightings are largely unknown. We took $g=1.12$. The atomic $\mathrm{S} / \mathrm{Fe}$ ratio in the CMC, $s_{\mathrm{cmc}}^{\prime}=0.52$, is just the solar value. Then the mixing fraction is given by

$$
\psi=\frac{g\left(s_{\mathrm{cmc}}^{\prime}-\zeta\right)}{\zeta(1-g)-s_{\mathrm{in}}^{\prime}+g s_{\mathrm{cmc}}^{\prime}} .
$$

Because $\psi$ is a decreasing function of $\zeta$, our lower limit on $\zeta$ gives an upper limit on $\psi$, so we find that

$$
\psi<0.65
$$

In other words, a mixing fraction $\psi>0.67$ would violate the measured lower limit on the $\mathrm{S} / \mathrm{Fe}$ ratio in the comet sample. This result depends on the assumption that most of the observed sulfur depletion predated or was concurrent with strong nebular mixing.

Subject to the assumptions discussed above, we can combine these two constraints to define a range of allowed inner nebula mixing fraction $0.50<\psi<0.65$. This fraction of inner solarsystem material in Wild2 implies that mixing was surprisingly efficient over large distances in the early solar nebula. This measurement may be used to constrain parameter space in mixing models of the solar nebula that provide quantitative predictions.

Bockelée-Morvan et al. (2002) used a two-dimensional turbulent radial mixing model of the protosolar nebula to compute the fraction of crystalline silicates as a function of heliocentric distance. They found that crystallinity at large distance is a strongly increasing function of the temperature of the nebula. Our measurement of a relatively large crystalline fraction is consistent with their "warm nebula" model. This model assumes a relatively large mass-accretion rate $\dot{M}_{0} \sim 10^{-5} M_{\odot}$ $\mathrm{yr}^{-1}$, and a relatively small dimensionless viscosity parameter $\alpha \sim 8 \times 10^{-3}$.

Ciesla (2007) has also developed a two-dimensional model of an evolving turbulent disk. This model exhibits a net outward flow along the midplane of the disk that can drive inner nebular materials to large distances. His model is also quantitative, so can be compared with the measurement presented here. For example, the models presented in Figures 3(A), (B) and (D) of Ciesla (2007) are inconsistent with our measurement, while that in Figure 3(C) is consistent with our crystalline fraction but probably not with our upper limit on $\psi$ based on the observed $\mathrm{S} / \mathrm{Fe}$ ratio.

Crystalline silicates have been observed in several longperiod (Oort Cloud) comets (Wooden et al. 2004) and in some Jupiter-family comets (Kelley et al. 2008). The diversity in the crystalline silicate fraction among comets is strong; some comets are strongly deficient in crystalline silicates (e.g., C/ 2006 P1 McNaught, Kelley et al. 2007), while others have crystalline silicate fractions up to $\sim 0.7$ (e.g., C/2001 Q4, Wooden et al. 2004). Silicates in disks of Herbig $\mathrm{Ae} / \mathrm{Be}$ stars show evidence for increasing crystallinity with age. This observation led Nuth et al. (2000) to suggest that the crystalline fraction in individual comets may serve as a clock to measure time of formation. A calibration of this clock either observationally or theoretically could allow for a estimation of the time of formation of $81 \mathrm{P} /$ Wild2.

Although there is general agreement that crystalline silicates could not have formed at the heliocentric distance of the Kuiper Belt, and so must have their origin in the inner nebula, it is not clear whether the site for crystallization is the hot inner ( $\leqslant$ 4 AU) nebula (Hersant 1994; Nuth 1999) or in interplanetary shocks at 2-10 AU at later times (Harker \& Desch 2002). Nuth \& Johnson (2006) have pointed out that the formation and transport of ferroan crystalline silicates from a hot inner nebula should be very inefficient because of their short lifetime against vaporization in such a region, so the presence of ferroan crystalline silicates in comets argues strongly in favor of shock processing. Assuming that the $\mathrm{Fe} / \mathrm{Si}$ abundance in Wild 2 is similar to CI, our measurement implies that $\sim 10 \%$ or more of the crystalline silicates are ferroan. Although Nuth \& Johnson (2006) did not treat this problem quantitatively, our measurement appears to support the shock model of crystal formation.

Our measurement also implies that comet Wild 2 may contain a significant fraction of relatively unprocessed molecular cloud material (see, however, Scott \& Krot 2005). The concentration of presolar grains may be an indication of primitiveness (Huss \& Draine 2006). 81P/Wild2 appears to be significantly depleted in presolar oxide grains as compared with the most primitive meteorites (McKeegan et al. 2008; Stadermann \& Floss 2008). This apparent paradox would be resolved if presolar oxides, which are typically $<300 \mathrm{~nm}$ in size, are preferentially lost in the corrosive $\mathrm{H}_{2} \mathrm{~S}$-rich cometary interior because of their chemical reactivity and small size (and, therefore, large surfaceto-volume ratio). This sulfidization reaction might happen either during the first $\sim 1 \mathrm{Myr}$ after accretion when the comet is warmed by radiogenic decay of ${ }^{26} \mathrm{Al}$ (Weidenschilling 1997; Choi et al. 2002), or perhaps in the contemporary warm subsurface reservoirs that feed the cometary jets. Laboratory studies of sulfidization of oxidized minerals may be able to address this question. If this is the case, one could expect that more chemically robust presolar grains, such as $\mathrm{SiC}$, may be present in Wild2 in concentrations comparable to those found in primitive meteorites or chondritic porous IDPs.

We are indebted to the referee of this paper, John Wasson. We are grateful to Don Brownlee, Dave Joswiak, Gary Huss, Buford Price, and Denton Ebel for helpful suggestions and discussions. This work was supported by NASA SPS and DDAP grants. We gratefully acknowledge the provision of XANES standard compounds by D. L. Bond, T. Borch, B. C. Bostick, T. Buonassisi, M. Burchell, C. S. Chan, S. Fendorf, C. Hansel, M. Heuer, A. Kearsley, C. S. Kim, M. Newville, P. Nico, 
P. O'Day, N. Rivera, K. Ross, C. Santelli, B. Toner, and G. A. Waychunas. The operations of the Advanced Light Source at Lawrence Berkeley National Laboratory are supported by the Director, Office of Science, Office of Basic Energy Sciences, U.S. Department of Energy under contract number DE-AC02$05 \mathrm{CH} 11231$.

\section{REFERENCES}

Alexander, C. M. O., Boss, A. P., \& Carlson, R. W. 2001, Science, 293, 64 Allègre, C., Manhès, G., \& Lewin, È. 2001, Earth \& Planet. Sci. Lett., 185, 49 Bland, P. A., Cressey, G., \& Menzies, O. N. 2004, Meteoritics Plan. Sci., 39, 3 Bockelée-Morvan, D., Gautier, D., Hersant, F., Huré, J.-M., \& Robert, F. 2002, A\&A, 2002, 1107

Boss, A. P., \& Graham, J. A. 1993, Icarus, 106, 168

Brearley, A. J., \& Jones, R. H. 1998, in Planetary Materials., ed. J. J. Papike Rev. Mineralogy, 36

Brownlee, D. E., et al. 2006, Science, 314, 1711

Bullock, et al. 2005, Geochim. Cosmochim. Acta, 60, 4253

Burchell, M. J., et al. 2008, Meteorit. Planet. Sci., 43, 23

Chernick, M. R. 1999, Bootstrap Methods: A Practitioner's Guide, Wiley Series in Probability and Statistics, (New York: Wiley)

Choi, Y.-J., Cohen, M., Merk, R., \& Prialnik, D. 2002, Icarus, 160, 300

Ciesla, F. J. 2007, Science, 318, 613

Davis, A. M, Grossman, L., \& Ganapathy, R. 1977, Nature, 265, 230

Flynn, G. F., et al. 2006, Science, 314, 1731

Gounelle, M., Spurný, P, \& Bland, P. A. 2006, Meteorit. Planet. Sci., 41, 135

Greenberg, J. M. 1998, A\&A, 330, 375

Hanner, M. S., et al. 1994, Icarus, 112, 490

Harker, D. E., \& Desch, S. J. 2002, ApJ, 565, L109

Hazemann, J. L., et al. 1992, Phys. Chem. Miner., 19, 25

Hersant, F., Gautier, D., \& Huré, J. 2001, ApJ, 554, 391

Huss, G. R., \& Draine, B. T. 2006, Proc. Int. Astron. Union, 2, 353

Irvine, W. M., et al. 2000, in Protostars and Planets IV, ed. V. Mannings, A. P. Boss, \& S. S. Russell (Tucson, AZ: Univ. Arizona Press), 1159

Ishii, H. A., et al. 2008, Science, 319, 447

Jarosewich, E. 1990, Meteoritics, 25, 323

Javoy, M. 1995, Geophys. Res. Lett., 22, 2219

Keller, L. P., et al. 2006, Nature, 417, 148

Kelley, M. S., et al. 2007, Bull. Am. Astron. Soc., 39, 827

Kelley, M. S., et al. 2008, in Asteroids, Comets, Meteors, Proc. LPI Conf. Ser., 8280

Kelly, S. D., Hesterberg, D., \& Ravel, B. 2008, Analysis of Soils and Minerals Using X-ray Absorption Spectroscopy, in Methods of Soil Analysis, Part
5-Mineralogical Methods, ed. A. L. Ulery \& L. R. Drees (Madison, WI: Soil Science Society of America), 367

Kemper, F., Vriend, W. J., \& Tielens, A. G. G. M. 2004, ApJ, 609, 826

Kraft, S., et al. 1996, Rev. Sci. Instrum., 67, 681

Krot, A. N., et al. 2000, in Protostars and Planets IV, ed. V. Mannings, A. P. Boss, \& S. S. Russell (Tucson, AZ: Univ. Arizona Press), 1019

Lodders, K. 2003, ApJ, 591, 1220

Lodders, K., \& Fegley, B. 1998, The Planetary Scientist's Companion (New York: Oxford Univ. Press), 371

Manceau, A., Marcus, M. A., \& Tamura, N. 2002, Rev. Mineral. Geochem., 49, 341

Marcus, M. A., et al. 2004, J. Synchrotron. Radiat., 11, 239

Marcus, M. A., et al. 2008a, Meteorit. Planet. Sci, 43, 87

Marcus, M. A., Westphal, A. J., \& Fakra, S. C. 2008b, J. Synchrotron. Radiat., 15,463

Mason, B., \& Wiik, H. B. 1962a, Am. Mus. Nov., 2115, 1

Mason, B., \& Wiik, H. B. 1962b, Am. Mus. Nov., 2106, 1

Mason, B., \& Wiik, H. B. 1966, Am. Mus. Nov., 2272, 1

Marty, B., et al. 2008, Science, 319, 75

McKeegan, K., et al. 2008, Science, 314, 1724

Nuth, J. A. 1999, Proc. 30th Lunar Planet. Sci. Conf., 1726

Nuth, J. A., Hill, H. G. M., \& Kletetschka, G. 2000, Nature, 349, 18

Nuth, J. A., \& Johnson, N. M. 2005, Icarus, 180, 243

O’Day, P. A., et al. 2004, Am. Mineral., 89, 572

Palme, H. 2001, Phil. Trans. R. Soc. A, 359, 2061

Palumbo, M. E., Geballe, T. R., \& Tielens, A. G. G. M. 1997, ApJ, 479, 839

Prinn, R. G. 1990, ApJ, 348, 725

Rubin, A. E., \& Wasson, J. T. 1995, Proc. 30th Met. Soc., 569

Ruzmaikina, T. V., \& Ip, W. H. 1994, Icarus, 112, 430

Scott, E. R. D., \& Krot, A. N. 2005, ApJ, 623, 571

Scheinost, A. C., et al. 2005, Phys. Scr., T115, 1038

Sekanina, Z. 2003, J. Geophys. Res. E, 108, 8112

Shu, F. H., Shang, H., \& Lee, T. 1996, Science, 271, 1545

Stadermann, F., \& Floss, C. 2008, Proc. 39th Lunar Planet. Sci. Conf., 1391

Tanaka, K. K., et al. 1998, Icarus, 134, 137

Urey, H. C., \& Craig, H. 1953, Geochim. Cosmochim. Acta, 4, 36

Wasson, J. T. 1985, Meteorites: Their Record of Early Solar-System History (New York: Freeman)

Wasson, J. T., \& Chou, C.-L. 1974, Meteoritics, 9, 69

Weidenschilling, S. J., et al. 1997, Icarus, 127, 290

Westphal, A. J., et al. 2004, Meteorit. Planet. Sci., 39, 1375

Westphal, A. J., et al. 2008, Meteorit. Planet. Sci., 43, 415

Wilke, M., et al. 2001, Am. Mineral., 86, 714

Wooden, D. H., et al. 2004, ApJ, 612, L77

Yanai, K. 1992, Proc. 23 Lunar Planet. Sci. Conf., 1559

Yin, Q.-Z. 2005, ASP Conf. Ser., Chondrites and the Protoplanetary Disk, 341, 632

Zolensky, M. E., et al. 2006, Science, 314, 1735 